\title{
Krank oder Simulant? Das Identifikationsproblem der Krankenkassen aus wirtschaftshistorischer Perspektive
}

\author{
Timothy W. Guinnane* \\ Yale University New Haven
}

Jochen Streb

Universität Mannheim

Manuel Frondel

Rheinisch-Westfälisches Institut für Wirtschaftsforschung, Essen und Ruhr-Universität Bochum

\section{Christoph M. Schmidt}

Rheinisch-Westfälisches Institut für Wirtschaftsforschung, Essen

Ruhr-Universität Bochum

\section{Einleitung}

Es gehört zum Selbstverständnis des modernen Sozialstaats, seine Bürger vor den schwerwiegendsten Folgen der Unwägbarkeiten des Lebens zu schützen. Im Mittelpunkt stehen dabei meist die wirtschaftlichen Konsequenzen: Die möglicherweise drastischen ökonomischen Auswirkungen von Krankheit und Behinderung, welche die von ihnen Betroffenen am vollen Einsatz im Arbeitsleben und somit am Erzielen von Einkommen hindern, stellen eines der herausragenden Motive für staatliches Eingreifen dar. Das wichtigste Instrument dafür sind Krankenversicherungssysteme ${ }^{1}$ unterschiedlichster Ausprägung, deren Design wie bei jeder anderen Versicherung auch - darauf zugeschnitten sein muss, den Versicherungsschutz und die negativen Anreizeffekte so gegeneinander abzuwägen, dass die Versicherung langfristig finanziell tragfähig ist. Krankenversicherungen

\footnotetext{
*Kontaktadressen: Prof. Timothy W. Guinnane, Department of Economics, Yale University, 27 Hillhouse Ave., New Haven, CT 06520-8269, E-Mail: timothy.guinnane@yale.edu; Prof. Dr. Jochen Streb, Universität Mannheim, L 7, 3-5, 68131 Mannheim, E-Mail: streb@uni-mannheim.de; Prof. Dr. Manuel Frondel, Rheinisch-Westfälisches Institut für Wirtschaftsforschung (RWI), Hohenzollernstr. 1-3, 45128 Essen, E-Mail: manuel.frondel@rwi-essen.de; Prof. Dr. Christoph M. Schmidt, Rheinisch-Westfälisches Institut für Wirtschaftsforschung (RWI), Hohenzollernstr. 1-3, 45128 Essen, E-Mail: schmidt@ rwi-essen.de.

1. Unsere Diskussion beschränkt sich auf diesen Zweig der sozialen Sicherung und die dort auftretenden Anreiz- und Informationsprobleme. Ähnlich gelagert sind die Motive der staatlichen Organisation der hier nicht tiefer analysierten Arbeitslosenversicherung, bei der im Prinzip die gleichen Anreiz- und Informationsprobleme auftreten. Der hier ebenfalls nicht weiter diskutierten Alterssicherung liegt ebenso ein solches Versicherungsmotiv zugrunde, gleichwohl das Erreichen des Pensionsalters kein überraschendes Schicksal, sondern (glücklicherweise) eine weitgehend zu erwartende Lebensphase darstellt, deren Herannahen für jeden erkennbar ist.
} 


\section{Timothy W. Guinnane, Jochen Streb, Manuel Frondel und Christoph M. Schmidt}

sind dabei von einem grundlegenden Erkenntnisproblem geplagt: Der - nur eingeschränkt möglich - korrekten Einschätzung des Gesundheitszustandes von Versicherten.

Im Prinzip würde man natürlich bei einem Krankenversicherungssystem sehr gerne ein Separationsgleichgewicht erreichen, bei dem tatsächlich Kranke unterstützt und Simulanten, die ihre Krankheit übertreiben oder gar vortäuschen, enttarnt werden. Das gilt insbesondere dann, wenn man eine Lohnfortzahlung im Krankheitsfall vorsieht. Sinn der Lohnfortzahlung ist, dass alle Kranken, die aufgrund ihrer Krankheit oder Behinderung nicht (voll) arbeitsfähig sind, sich die nötige Zeit nehmen können, um sich auszukurieren. Auf diese Weise sollen langfristige Folgen, wie dauerhafte Arbeitsunfähigkeit und Invalidität, die bei einer Verschleppung gesundheitlicher Probleme drohen, vermieden werden, ohne dass die Betroffenen erhebliche finanzielle Einbußen hinnehmen müssen, wie sie bei einer Abwesenheit vom Arbeitsplatz drohen.

Allerdings werden durch diesen Schutz vor wirtschaftlichen Konsequenzen in unvermeidbarer Weise Verhaltensanreize ausgelöst. So könnten Arbeitnehmer, die ihre Krankheit bereits überwunden haben oder die nur geringfügig oder überhaupt nicht unter einem gesundheitlichen Problem leiden, durch die finanziellen Anreize der Lohnfortzahlung durchaus zum Fernbleiben vom Arbeitsplatz ermutigt werden. Allerdings ist die Balance zwischen Simulation und Verschleppung in einem System der Lohnfortzahlung keineswegs eindeutig. Denn trotz der durch die Lohnfortzahlung gebotenen Anreize könnten sich Arbeitnehmer möglicherweise gezwungen sehen, sich gegen ihre Gesundheit und für den Arbeitsplatz zu entscheiden, falls sie bei einem krankheitsbedingten Fernbleiben vom Arbeitsplatz dessen Verlust fürchten.

Das weitgehende Fehlen der objektiven Messbarkeit des Gesundheitszustands behindert den Aufbau eines trennscharfen Systems der Lohnfortzahlung im Krankheitsfall: Obwohl nach dem Kenntnisstand der modernen Medizin gesundheitliche Probleme vielfach korrekt diagnostiziert werden, entziehen sich entscheidende Beeinträchtigungen des individuellen Wohlbefindens, insbesondere Schwächegefühle und Schmerz, einer objektiv nachvollziehbaren Messung. Das Wissen um diese mangelnde Trennschärfe vorausgesetzt, besteht für Versicherte in jedem System der Lohnfortzahlung im Krankheitsfalle ein mehr oder weniger großer Anreiz („Moral Hazard“), dessen Vorzüge auf dem Wege der Simulation gesundheitlicher Probleme auszunutzen.

Daher verwundert es nicht, dass Diskussionen zum Krankenstand in einzelnen Wirtschaftzweigen oder gar der gesamten Volkswirtschaft immer wieder mit hoher Emotionalität geführt werden. Dies gilt nicht zuletzt für die Schwankungen im gesamtwirtschaftlichen Krankenstand, die im Zuge konjunktureller Hoch- und Tiefphasen regelmäßig zu beobachten sind. Beispielsweise zeigen Schnabel und Stephan (1993) mit Hilfe eines Zeitreihenmodells für Westdeutschland, dass zwischen 1965 und 1991 ein signifikant positiver Zusammenhang zwischen der Höhe des jährlichen Krankenstands und der Veränderungsrate des realen Bruttoinlandsprodukts bestand.

Dieser Befund kann auf unterschiedliche Arten interpretiert werden: Nutzten Arbeitnehmer, wie so oft vermutet (Barmby und Treble 1991), die erhöhte Arbeitsplatzsicherheit in konjunkturellen Hochphasen zu vermehrter Simulation? Oder ist es vielleicht genau umgekehrt (Fahr und Frick 2007), dass Arbeitnehmer während einer wirtschaftlichen Depression aus Angst vor einer Entlassung trotz 
vorliegender Krankheit am Arbeitsplatz erscheinen? Neben diesen Phänomenen des Absentismus bzw. Präsentismus ist drittens auch denkbar, dass während eines konjunkturellen Booms die Arbeitsbelastung und damit auch Krankheitsanfälligkeit und Unfallwahrscheinlichkeit ansteigen, was eine Zunahme des Krankenstands in Phasen der Hochkonjunktur ergeben könnte. ${ }^{2}$

Ein wirtschaftshistorisches Forschungsprojekt, das durch den außeruniversitären Zweig der Exzellenzinitiative gefördert wurde, zeigt, dass „Simulantentum“ ein nicht erst seit Mitte des 20. Jahrhunderts wahrgenommenes Problem ist. ${ }^{3}$ Vielmehr wurde dies schon im 19. Jahrhundert mit großer Vehemenz von den knappschaftlichen Krankenversicherungen, in denen Bergleute versichert waren, beklagt. Während Guinnane und Streb (2011) sowie Bluma, Schulz und Streb (2010) die wesentlichen empirischen Ergebnisse dieses Forschungsprojektes bezüglich der Simulationsprobleme von Knappschaften zusammenfassen, ist es das Ziel des vorliegenden Artikels, aufzuzeigen, dass es aus methodischer Sicht gerade diese wirtschaftshistorische Perspektive ist, die einen wertvollen Beitrag zur gesundheitsökonomischen Literatur ermöglicht.

Darüber hinaus verdienen die Knappschaften auch aus inhaltlichen Gründen noch heute das Interesse der Wirtschaftswissenschaften, weil sie bereits Mitte des 19. Jahrhunderts, und damit dreißig Jahre vor der Einführung der Bismarckschen Sozialversicherungsgesetzgebung, eine berufsständische Sozialversicherung gegen Krankheit und Invalidität etablierten, die wesentliche Charakteristika unseres heutigen Sozialversicherungssystems vorwegnahm. So deckten bereits vor 150 Jahren die knappschaftlichen Krankenversicherungen die Kosten für Arzneimittel, ärztliche Behandlung und Krankenhausaufenthalte und gewährten zusätzlich einen Krankenlohn pro Krankentag als Lohnersatzleistung. ${ }^{4}$

Ebenso wie heute wurden diese Ausgaben im Umlageverfahren finanziert, in dem die zwangsversicherten Bergleute und die Bergwerksunternehmer regelmäßig Beiträge entrichteten, wobei vor 1907 die Beiträge nicht zwangsläufig zu jeweils 50 Prozent geschultert wurden (Jopp 2011a). Teilt man die Einschätzung, die knappschaftliche Versicherung hätte maßgeblichen Vorbildcharakter für die Bismarcksche Sozialversicherungsgesetzgebung gehabt (Hennock 2007), so kommt man nicht umhin, die Bedeutung der Knappschaft als Modell für alle jene Volkswirtschaften $\mathrm{zu}$ reklamieren, die eher in der Bismarckschen Sozialversicherungstradition denn in der angelsächsischen Tradition der Grundsicherung stehen.

2. Zum Problem des so genannten Präsentismus aus betriebswirtschaftlicher Perspektive vgl. Iverson, Krause (2007). Darüber hinaus wird auch in der betriebswirtschaftlichen Literatur das Problem der Simulation von Krankheit häufig diskutiert. Hier steht im Mittelpunkt der Debatte, wie durch eine Intensivierung der Kommunikation mit überdurchschnittlich oft erkrankten Arbeitnehmern in Form so genannter Rückkehrgespräche, aber auch mit Ärzten, die durch häufige Krankschreibungen auffallen, das Problem der Simulation wirksam bekämpft werden kann (Olesch 1993, Spies und Beigel 1996, Borowiak und Taubert 1997).

3. Im Rahmen des Pakts für Forschung und Innovation der Leibniz-Gemeinschaft wurde von 2007 bis 2009 das interdisziplinäre Forschungsprojekt „Vergangenheit und Zukunft sozialer Sicherungssysteme am Beispiel der Bundesknappschaft und ihrer Nachfolger" gefördert. Einen Überblick über die insgesamt fünf Teilprojekte gibt Bartels et al. (2009).

4. Das Entgeltfortzahlungsgesetz vom 1. Januar 1970 übertrug die Pflicht zur Zahlung eines Krankengelds von den Krankenkassen auf die Arbeitgeber. Folgerichtig beklagen sich seither weniger die Krankenkassen als vielmehr die Arbeitgeber(verbände) über die Simulation der Arbeitnehmer. 


\section{Timothy W. Guinnane, Jochen Streb, Manuel Frondel und Christoph M. Schmidt}

Sollten sich bei diesen Vorgängern der heutigen Krankenversicherungen, die, wie im Folgenden noch ausgeführt wird, durch intensive soziale Kontrolle geprägt waren, erhebliche Probleme des Moral Hazard nachweisen lassen, so dürfte es schwer sein, die aktuelle Diskussion um das Problem des simulierten Krankenstands als einen fern der Lebenswirklichkeit liegenden Gedankengang der ökonomischen Theorie abzutun. Die hier dargestellten empirischen Ergebnisse vermitteln daher Einsichten, die auch in aktuellen gesundheitspolitischen Debatten hilfreich sein können.

Im Folgenden wird mit Rückgriff auf die aus dem wirtschaftshistorischen Forschungsprojekt resultierenden Erkenntnisse belegt, dass man bereits im 19. Jahrhundert die ökonomischen Implikationen von Moral Hazard in der Krankenversicherung sehr genau verstand und mit Hilfe verschiedener Anreizund Kontrollmechanismen zu bekämpfen suchte (Abschnitt 2). Sodann werden wir zeigen, dass sich die historischen Knappschaftskrankenkassen aufgrund ihrer Gebietsmonopole und ihres verhältnismäßig großen geschäftspolitischen Handlungsspielraums in besonderem Maße zur empirischen Analyse von MoralHazard-Problemen eignen (Abschnitt 3). Wichtigstes Resultat der in Abschnitt 4 zusammengefassten empirischen Ergebnisse ist, dass von der Höhe und Dauer des Krankenlohns je Krankentag ein starker Anreiz zur Simulation von Krankheit ausging. Der letzte Abschnitt zieht die Schlussfolgerung, dass die hier eingenommene wirtschaftshistorische Perspektive in weitaus größerem Maße zur Beantwortung von aktuellen Fragestellungen genutzt werden kann, als allein zur Analyse von Moral Hazard bei Krankenversicherungen.

\section{2. „Simulantentum“ ist ein altbekanntes Problem}

Da Versicherungen so grundlegend von Anreizproblemen geplagt werden, hat sich eine umfangreiche ökonomische Literatur damit beschäftigt, wie diese eingeschränkt werden können. Mit Anreiz- und Kontrollmechanismen stehen den Konstrukteuren von Systemen der Lohnfortzahlung im Krankheitsfall zwei prinzipielle Wege offen, um dem Problem der mangelhaften Beobachtbarkeit des Gesundheitszustands eines Versicherten entgegenzuwirken. So können zum einen die Bedingungen, unter denen es zu einer Lohnfortzahlung kommt, so angepasst werden, dass das Ausnutzen des Systems durch Simulation vergleichsweise unattraktiv ist.

Das vielleicht herausragende Beispiel für derartige Regelungen ist die Wiedereinführung einer Anzahl von Karenztagen, wie sie beispielsweise von Boss (2000) gefordert wird: Lohnfortzahlung im Krankheitsfalle wird zwar gewährt, aber erst, nachdem der Krankgemeldete eine festgelegte Wartezeit durchlaufen hat. Schnabel und Stephan (1993) beobachten, dass der Krankenstand innerhalb einer Arbeitswoche in der Regel einen u-förmigen Verlauf aufweist, mit Höchstständen jeweils am Montag und Freitag. Wenn diese Verteilung vorrangig dem Umstand geschuldet ist, dass Arbeitnehmer durch Vortäuschen einer kurzfristigen Krankheit ihr Wochenende zu verlängern suchen, dürfte eine Karenzzeit tatsächlich eine wirksame Maßnahme zur Bekämpfung dieser Art von Simulation sein.

Die Anreizwirkungen von Karenztagen wurde auch von den Knappschaften des 19. Jahrhunderts erkannt, welche teilweise Wartezeiten von bis zu 3 Tagen eingeführt hatten (Guinnane und Streb 2011). Der Bekämpfung von Simulantentum diente auch der von einigen Knappschaften eingeführte 
Hospitalzwang für alle krankgemeldeten Bergleute. Einerseits verlor die durch Simulation erworbene arbeitsfreie Zeit deutlich an Attraktivität, wenn sie im Krankenhaus verbracht werden musste, zumal dann, wenn während der Dauer des Krankenhausaufenthalts das Krankengeld nicht oder nur teilweise ausgezahlt wurde. Andererseits fiel es im Krankenhaus vielleicht auch leichter, Simulation durch Langzeitbeobachtung nachzuweisen.

Die Zahlen sprechen für einen Erfolg des Hospitalzwangs. So wies der Oberschlesische Knappschaftsverein, der etwa 70 Prozent der arbeitsunfähigen Bergleute im Krankenhaus versorgte, zwischen 1901 und 1908 durchschnittlich nur 6,4 Krankentage je Mitglied auf, während sich diese Kennziffer für die übrigen preußischen Knappschaftskrankenkassen auf 10,8 Tage und für sämtliche Krankenkassen des Deutschen Reichs auf 7,6 Tage belief (Lauf 2006). Die Idee, Simulanten durch den Hospitalzwang zumindest einen Teil der mit dem Fortbleiben vom Arbeitsplatz verbundenen Lebensqualität zu nehmen, erinnert an das Verbot des Pub-Besuchs bei Krankheit, das einige englische Friendly Societies verfolgten, welche in mancherlei Hinsicht mit den Knappschaften vergleichbar waren. ${ }^{5}$

Ein anderer grundlegender Weg zur Erreichung des angestrebten Separationsgleichgewichts zwischen Kranken und Simulanten ist die verbesserte Informationsgewinnung. Hierzu werden in der Regel gesetzlich beziehungsweise ethisch zu Wahrhaftigkeit verpflichtete oder vertraglich an den Versicherer gebundene Ärzte in die Bewilligung der Lohnfortzahlung eingebunden. Dieser Lösungsansatz hat allerdings seine Grenzen: Schon die Zeitgenossen im 19. Jahrhundert vermuteten, dass Ärzte im Zweifelsfall lieber zugunsten der Arbeitnehmer irrten und selbst vermeintlich recht offensichtliche Simulation deckten. ${ }^{6}$ Die Knappschaften reagierten auf solcherlei Verdacht durch die Einführung zusätzlicher Kontrollorgane wie der Etablierung von Vertrauensärzten und des Sprengelarztsystems, in dem das Arzthonorar nicht fallabhängig, sondern als Pauschale gewährt wurde. Diese Pauschale hing nur von der Zahl der Versicherten im Einzugsgebiet des Sprengelarztes ab. ${ }^{7}$

Die Interessenharmonie zwischen Ärzten und Patienten mag im aktuellen Gesundheitswesen sogar verstärkt vorliegen, da Ärzte sich möglicherweise nicht zum Anwalt von Arbeitgeberinteressen machen wollen, aber auch, weil der immer härtere Wettbewerb um Patienten es den Ärzten schwer macht, den Wunsch nach einer Krankschreibung zu verweigern. Es dürften gerade Simulanten sein, die einen solchen Wunsch am vehementesten vortragen.

Systeme der sozialen Sicherung können jedoch auch auf interne Regulierungsmechanismen zurückgreifen, die auf sozialem Druck basieren. Es liegt auf der Hand, dass ein sozialverträgliches Verhalten der Mitglieder einer Gemeinschaft besonders dann durch wechselseitige Kontrolle erzwungen werden kann, wenn die Gemeinschaft vergleichsweise homogen und von überschaubarer Größe ist. In der heutigen Zeit, in der Krankenversicherungen typischerweise Hunderttausende von Mitgliedern verschiedensten Alters mit unterschiedlichen

5. Zu den Britischen Friendly Societies vgl. z.B. Riley (1997).

6. So schrieb Oberbergrat Adolf Arndt (1894, S. 133f.): „Bei der jetzt vorhandenen Überzahl an Ärzten und bei deren nicht seltenem Mangel an ausgiebiger Praxis werde es den Arbeitern nicht schwer, solche Personen zu Ärzten zu wählen, welche den an sie gerichteten Wünschen zu willfahren geneigt seien und leicht Krankenscheine ausstellen."

7. Zur Kontrollspirale in den Knappschaften vgl. Bluma, Schulz, Streb (2010). 


\section{Timothy W. Guinnane, Jochen Streb, Manuel Frondel und Christoph M. Schmidt}

beruflichen Tätigkeiten aufweisen und sich die wenigsten Mitglieder kennen, ist dieser Mechanismus zur Erreichung sozialverträglichen Verhaltens weitgehend in den Hintergrund getreten. ${ }^{8}$

$\mathrm{Zu}$ Zeiten der Knappschaften war dies noch anders. So entsprach es dem Selbstverständnis der Knappschaftsvereine, dass die in ihnen organisierten Bergleute eine privilegierte, von ehrenhaftem Geist und tiefer Solidarität geprägte Gemeinschaft bildeten. Arbeitnehmer und Arbeitgeber waren bei der Organisation dieser Versicherungssysteme gegen Unfälle, Krankheit und Invalidität gleichermaßen beteiligt und - im Rahmen der geltenden gesetzlichen Regelungen - für zentrale Charakteristika wie Fortzahlungsdauer und -höhe selbst verantwortlich. Unter solchen Umständen fiel es dem Einzelnen aller Vermutung nach vergleichsweise schwer, durch Simulation Vorteile zu erringen.

Es liegt nahe, dass Moral Hazard in der heutigen, weit anonymeren Welt des Krankenversicherungswesens wohl eine viel größere Rolle spielt, als zu Zeiten der Knappschaften, obwohl bereits diese nach eigener Einschätzung von Moral Hazard geplagt wurden. Dem Nachteil der Anonymität steht der Vorteil gegenüber, dass es gerade die heute übliche Größe von Krankenversicherungen erlaubt, Verwaltungskosten auf viele Köpfe zu verteilen und eine breite Streuung von Risiken zu erreichen. Die Kosten der Versicherung sollten so für die einzelnen Versicherten erheblich reduziert werden können. Da dies wohl unverzichtbar ist, stellt sich die Frage, ob es überhaupt wirksame Mechanismen gibt, um Problemen des Moral Hazard entgegenwirken zu können.

\section{Knappschaften als ideales Studienobjekt}

Die beiden hier aufgeworfenen Fragen, ob erstens Moral Hazard in Krankenversicherungs- und Lohnfortzahlungssystemen eine erhebliche Rolle spielt und ob es zweitens wirksame Mechanismen gibt, um Moral Hazard erfolgreich bekämpfen zu können, sind auf Basis aktuellen Datenmaterials aus mindestens zwei Gründen nur sehr schwer zu beantworten. Zum einen gibt es bekanntlich eine adverse Selektion von Versicherten bezüglich ihrer gesundheitlichen Risiken, so dass unterschiedliche Krankenkassen nur schwer miteinander vergleichbar sind. Im Gegensatz zu den Knappschaften dürften Krankenversicherungen darüber hinaus äußerst heterogen in ihrer Mitgliederstruktur sein, welche zudem über die Zeit hinweg stark variiert. Darüber hinaus sind die zu untersuchenden Regelungen vermutlich auch mit der aktuellen Lage am Arbeitsmarkt systematisch verknüpft. Fahr und Frick (2007) zeigen, dass es starker Identifikationsannahmen bedarf, um in diesem Kontext Belege für die Bedeutung von Moral Hazard zu sammeln.

Zum anderen wird man selbst in Situationen, in denen es gelingt, eine vergleichsweise homogene Grundgesamtheit im Hinblick auf ihre krankheitsbedingte Abwesenheit vom Arbeitsplatz zu untersuchen, in den zentralen Parametern, bei denen man einen Einfluss auf die Intensität des Moral Hazard vermuten kann, nur eine recht dürftige Variation beobachten können. So werden

8. Natürlich dürfte es am Arbeitsplatz unangenehm auffallen, wenn ein Einzelner beispielsweise immer wieder nach Feiertagen oder bei so genannten Brückentagen mit vermeintlichen Rückenproblemen ausfällt. Aber angesichts der üppigen Ausstattung mit Urlaubsansprüchen, die für den deutschen Arbeitsmarkt typisch ist, dürften gerade notorische Simulanten geschickt genug sein, um ihr Verhalten nicht derart offensichtlich werden zu lassen. 
die relativen Lohnersatzleistungen im Krankheitsfalle für die Individuen des Sozio-ökonomischen Panels (SOEP) im gesamten Beobachtungszeitraum weitgehend unverändert sein. Hierdurch wird der Vorzug, durch eine weitreichende Einbeziehung von Kontrollvariablen analytische Homogenität zu gewährleisten, zunichte gemacht. Insbesondere ist es nur sehr schwer vorstellbar, in aktuellen Daten Variationen der informellen Kontrollmechanismen vorzufinden, die mehr oder weniger direkte Rückschlüsse auf ihre Wirksamkeit zur Eindämmung von Moral Hazard zulassen würden.

Die wirtschaftshistorische Analyse der Determinanten des Krankenstands in den Knappschaftskrankenkassen des 19. Jahrhunderts bietet eine recht einmalige Chance, diese beiden Hemmnisse zu überwinden, um zu validen Aussagen auf Basis einer empirischen Analyse zu kommen: Erstens handelt es sich bei Knappschaften um Organisationen mit einer äußerst homogenen Mitgliederschaft. Darüber hinaus war die Versicherung in der jeweils zuständigen Knappschaft für alle im Bergbau tätigen Arbeitnehmer verpflichtend. Somit konnte adverse Selektion gar nicht in dem Maße auftreten, wie es bei einer auf Freiwilligkeit und Wahlfreiheit beruhenden Krankenversicherung der Fall gewesen wäre. Ganz perfekt ist jedoch auch diese Datengrundlage nicht, denn im Laufe der Zeit erlebten einige Zechen ihren Niedergang. Typischerweise war die Belegschaft in diesen Zechen vergleichsweise alt und man könnte vermuten, dass diese einen höheren Krankenstand aufgewiesen hat als die Belegschaft prosperierender Zechen. Tatsächlich aber setzten die Bergwerksunternehmer ältere Bergleute vorwiegend zu ungefährlicheren und weniger gesundheitsschädlichen Tätigkeiten über Tage ein, so dass die Altersstruktur der Belegschaft insgesamt keinen signifikanten Einfluss auf den Krankenstand nahm.

Zweitens waren die Knappschaften in ihrer Geschäftstätigkeit auf bestimmte Regionen oder gar Unternehmen begrenzt und agierten innerhalb ihres Gebietsmonopols ohne Wettbewerbsdruck durch andere Knappschaften, die dort keine Mitglieder werben durften. Hierdurch ergab sich für jede einzelne der von uns untersuchten Knappschaften ${ }^{9}$ der versicherungsökonomische Handlungsspielraum, die Höhe der Beiträge und Leistungen sowie ihre Maßnahmen zur Bekämpfung von Simulation im Rahmen der allgemeinen gesetzlichen Vorgaben nach eigenem Ermessen zu gestalten. Für die empirische Analyse bedeutet dies, dass wir über alle Knappschaften hinweg eine erhebliche Varianz der zentralen Einflussvariablen bei sonst gleichen Rahmenbedingungen beobachten können. Dies ist ein entscheidender Vorteil gegenüber internationalen Vergleichen von Sozialversicherungssystemen, bei denen einerseits zwar Varianz in den zentralen Einflussvariablen auftritt, aber andererseits auch in den gesetzlichen, ökonomischen oder kulturellen Rahmenbedingungen. ${ }^{10}$

Drittens können wir in dieser wirtschaftshistorischen Betrachtung einen vergleichsweise langen Zeitraum analysieren, in dem zahlreiche gesetzliche

9. In Preußen erreichte die Zahl der unabhängigen Knappschaften ihren Höchststand von $91 \mathrm{im} \mathrm{Jahr}$ 1871, in Bayern von 41 im Jahr 1896. Vor und während des Ersten Weltkriegs kam es in beiden Ländern durch Fusionen und Schließungen zu einem Konzentrationsprozess. Im Jahr 1923 wurde die Reichsknappschaft gegründet, die alle noch vorhandenen Einzelknappschaften aufnahm. Vgl. Jopp (2010, 2011a, 2011b).

10. Für einen internationalen Vergleich von aktuellen Krankenversicherungssystemen vgl. z.B. ScheilAdlung und Sandner (2010). Für einen Vergleich der europäischen Krankenversicherungen im späten 19. Jahrhundert vgl. Murray (2005). 


\section{Timothy W. Guinnane, Jochen Streb, Manuel Frondel und Christoph M. Schmidt}

Regelungen eingeführt oder geändert wurden. Mit den Veränderungen in Bergbaurecht und -verwaltung zur Mitte des 19. Jahrhunderts waren die Knappschaften gezwungen, eine gewaltige Transformation zu vollziehen: Von kleineren, oft informell geführten Organisationen mussten die Knappschaften zu professionellen Einrichtungen heranwachsen, die den schwierigen Balanceakt meistern konnten, für ihre Mitglieder ein ansprechendes Netz der sozialen Sicherung aufzuspannen, ohne dass Moral Hazard ihre Bemühungen um Fairness zunichte machte. Dabei nahm ihre Größe sowohl durch internes Wachstum in den prosperierenden Revieren zu als auch durch Übernahmen und Zusammenlegungen. In der zweiten Hälfte des neunzehnten Jahrhunderts änderten sich überdies die gesetzlichen Anforderungen an die Höhe und Dauer der finanziellen Unterstützung erheblich. Damit wurden diejenigen Einflussfaktoren variiert, die von den Knappschaften selbstverantwortlich gesetzt wurden.

Auf diese Weise lässt sich der Zusammenhang zwischen der Größe einer Knappschaft und dem Vorliegen von "Simulantentum" anhand der mehrere Jahrzehnte umspannenden Daten empirisch testen. Es liegt nahe, dass Bergleute in einer größeren Knappschaft weniger Skrupel hatten, das System durch Simulation auszunutzen, da die Geschädigten für sie wohl weitgehend anonym blieben. So war es bei größeren oder räumlich weit auseinander gezogenen Knappschaften nahezu ausgeschlossen, dass die Mehrzahl der Mitglieder (regelmäßig) zusammentraf. Im Gegensatz dazu dürften Bergleute in kleineren Einheiten nicht nur alle ihre Kollegen gekannt haben, sondern auch zu einem Urteil darüber in der Lage gewesen sein, wie glaubhaft eine krankheitsbedingte Abwesenheit einzuschätzen war.

Auf der Basis des vorliegenden Datenmaterials ist es außerdem möglich, den Zusammenhang zwischen der Generosität der Lohnfortzahlung und dem Auftreten von Moral Hazard empirisch zu testen. Für die Analyse besonders förderlich ist die Homogenität der Rahmenbedingungen. Aus empirischer Sicht hilfreich ist auch, dass Leistungserhöhungen nicht nur von den Knappschaften selbst beschlossen wurden, sondern auch vom Gesetzgeber auferlegt wurden, sodass diese Variation im Sinne der Kausalanalyse als exogener Impuls angesehen werden kann, nicht als endogene Reaktion der Knappschaftsverwaltungen. So waren die Knappschaften durch ihre Eingliederung in das Bismarcksche Sozialversicherungssystem gezwungen, zweimal die Anreize zur Simulation deutlich zu verstärken: Ab 1887 und dann erneut ab 1905 mussten sie die Höhe ihrer Krankengeldzahlungen und insbesondere deren Dauer deutlich erhöhen, auf zuerst 13, dann auf 26 Wochen.

Schließlich erlauben es diese historischen Daten auch, den Einfluss des sektoralen Konjunkturzyklus auf den Krankenstand zu analysieren. So vertreten aktuelle Studien oft die Auffassung, dass Arbeitnehmer in konjunkturellen Krisen Krankschreibungen vermeiden, da sie sich vor einer Entlassung fürchten. Der Einfluss der konjunkturellen Lage auf den Krankenstand wäre dann positiv: Je besser die Wirtschaftslage, umso stärker dürfte das Ausmaß an Simulation ausfallen. Interessanterweise vermuteten die Zeitgenossen des 19. Jahrhunderts aber eher einen negativen Zusammenhang: „Wenn die Löhne fielen, namentlich aber, wenn die Arbeiter nicht mehr ganze, sondern nur noch theilweise Schichten verführen, steigen erfahrungsgemäß die Krankheiten, und damit die Belastung der Knappschaftskasse. Es kommt sogar in Zeiten absteigender Konjunktur vor, dass einzelne Arbeitgeber ihre Arbeiter, um den Lohn zu sparen, aufforderten sich krank zu melden, also zu simulieren" (Arndt 1894, 134). In diesem Fall nutzten die Arbeitgeber die knappschaftliche 
Tabelle 1 Mittelwerte und Standardabweichungen der wichtigsten Variablen

\begin{tabular}{lcc}
\hline Variable & Mittelwert & Standardabweichung \\
\hline Krankentage & 7,42 & 4,54 \\
Krankenlohn pro Krankentag (in Mark) & 0,95 & 0,63 \\
Mitgliederzahl & 4060 & 17070 \\
Arbeitgeberanteil an den gesamten KV-Kosten & 0,43 & 0,11 \\
Mitglieder pro Betrieb & 320 & 570 \\
Kohleproduktion je Oberbergamtsbezirk & 14,09 & 11,91 \\
$\quad$ (in Mio. Tonnen) & 22,20 & 28,75 \\
Arbeitgeberbeitrag pro Mitglied (in Mark) & 0,10 & 0,11 \\
Rheumatismusfälle pro Mitglied & 0,06 & 0,05 \\
Fälle von Prellungen je Mitglied & &
\end{tabular}

Mittelwerte und Standardabweichungen wurden über alle Beobachtungsjahre und Knappschaften berechnet.

Krankenversicherung implizit als Arbeitslosenversicherung, welche in Deutschland erst am Ende der Weimarer Republik eingeführt wurde.

\section{Ergebnisse einer empirischen wirtschaftshistorischen Studie}

Die Fülle und Vielfalt der von der preußischen (und zum Teil auch von der bayerischen) Knappschaftsstatistik bereitgestellten Daten erlaubt es, auf Grundlage von Knappschaft-Jahr-Durchschnitten für die Jahre 1867-1907 zu untersuchen, welche der theoretisch denkbaren Einflussfaktoren den Krankenstand in den Knappschaftskrankenkassen tatsächlich beeinflussten. ${ }^{11}$ Der Krankenstand wird im Folgenden durch die durchschnittliche Zahl der Krankentage pro Mitglied einer Knappschaft in einem Jahr gemessen, welche im Mittel über den gesamten Untersuchungszeitraum (1867-1907) bei rund 7,5 Tagen lag (Tabelle 1). Ein wichtiger Erklärungsfaktor dafür ist die Höhe der Lohnersatzleistung, mithin der Krankenlohn pro Krankentag, der sich als Quotient aus den gesamten Krankengeldzahlungen während eines Jahres und der Zahl der Krankentage errechnen lässt.

\subsection{Krankentage pro Mitglied}

Theoretisch gehen vom Krankenlohn je Krankentag zwei entgegengesetzte Wirkungen aus: Einerseits wuchsen mit steigendem Krankenlohn je Krankentag die Anreize der Bergleute zur Simulation. Andererseits mag ein (gesetzlich vorgegebener) Anstieg des Krankenlohns je Krankentag aber auch dazu geführt haben, dass sich die Knappschaftsverwaltungen angesichts des steigenden Kostendrucks stärker um die Aufdeckung von Simulation und damit eine Verringerung des Krankenstands bemühten. Welcher dieser Effekte im historischen Einzelfall überwog, ist a priori unbestimmt und kann nur durch die quantitative Analyse aufgedeckt werden.

Als zusätzliche Schwierigkeit kommt bei dieser Analyse hinzu, dass der Krankenlohn pro Krankentag endogen sein könnte, weil Knappschaften, die 


\section{Timothy W. Guinnane, Jochen Streb, Manuel Frondel und Christoph M. Schmidt}

sich einem hohen Krankenstand gegenüber sahen, versucht waren, diesen durch eine Kürzung der Lohnersatzleistung zu reduzieren. ${ }^{12}$ Zur Lösung dieses Endogenitätsproblems haben wir in den hier dargestellten Modellen den Beitrag der mutmaßlich endogenen Variable „Krankenlohn pro Krankentag“ mit Hilfe der Instrumentvariablen „Arbeitgeberbeitrag pro Mitglied“ geschätzt. Dass der Koeffizient des instrumentierten Krankenlohns je Krankentag als direkter Ausdruck von mit der Höhe der Lohnersatzleistung verbundenen Moral-Hazard-Problemen $\mathrm{zu}$ verstehen ist, ist die zentrale Identifikationsannahme dieser Analyse.

Der Arbeitgeberbeitrag zur Knappschaftsversicherung je Mitglied ergibt sich aus sämtlichen Kosten einer Knappschaft und ist somit nicht allein das Resultat der Krankengeldzahlungen, sondern auch der medizinischen und anderer Kosten sowie der Pensionslasten. Dieser Arbeitgeberbeitrag je Mitglied, welcher offenkundig zwar mit der endogenen Variablen „Krankenlohn pro Krankentag“ korreliert ist, erscheint als Instrument sinnvoll, da dieser Beitrag weniger das Ergebnis des Krankenstands, sondern vor allem das Resultat der Lohnverhandlungen zwischen Arbeitgebern und den Bergleuten gewesen sein sollte. War die Arbeitsangebotssituation angespannt, waren die Zechenbetreiber wohl eher bereit, höhere Beiträge zu den Knappschaftsversicherungen zu bezahlen als zu anderen Zeiten.

Um für die vom Reichsgesetzgeber exogen vorgegebene zweimalige Erhöhung der Höhe und Dauer des Krankenlohns je Krankentag zu kontrollieren, werden zwei Dummyvariablen verwendet, die für die Zeiträume „1887 und später“ beziehungsweise „1905 und später" den Wert 1 annehmen. Außerdem berücksichtigen wir die Knappschaftsgröße, da die soziale Selbstkontrolle der Bergleute umso weniger funktionieren dürfte, je höher die „Mitgliederzahl“ der Knappschaft und je größer die Anzahl der „Mitglieder pro Betrieb“ ausfallen. Durch die Berücksichtigung dieser beiden Faktoren wird unmittelbar der von den Zeitgenossen so hervorgehobene positive Zusammenhang zwischen Knappschaftsgröße und Krankenstand untersucht. Um für sektorale konjunkturelle Effekte zu kontrollieren, benutzen wir die jährliche „Kohleproduktion je Oberbergamtsbezirk“, in dem die betreffende Knappschaft tätig war. ${ }^{13}$

Tabelle 2 zeigt die Ergebnisse der zweistufigen Instrumentvariablenschätzung. Betrachten wir die zweite Stufe der IV-Schätzung, so können wir festhalten: ${ }^{14}$ Erstens

12. Wilhelm Bülow (1905, S. 64) beschreibt eine solche Reaktion bereits für die Zeit unmittelbar nach der Einführung einer neuen Knappschaftsverordnung in den Bergamtsbezirken Bochum und Essen im Jahr 1924: „Es zeigte sich bald nach der Einführung des neuen Reglements, dass die Vermögen der Knappschaftskassen keinen merklichen Zuwachs mehr erfuhren, indem die Ausgaben die Einnahmen fast völlig aufzehrten. Dies hatte seine Ursache wesentlich darin, [...] dass die Krankenschichtlöhne zu hoch angesetzt waren, wodurch zu manchen Simulationen und Verschleppungen von Krankheiten Anlass gegeben wurde. Eine oberbergamtliche Verfügung betr. Verhütung von Simulationen, nach der das Krankengeld erst vom fünften Tage ab und nach erfolgter Konstatierung der Arbeitsunfähigkeit durch den Bergarzt verabreicht werden durfte, schuf keine Besserung. Erst als am 31. Juli 1834 die Krankenlöhne auf 2/3 des früheren Betrages heruntergesetzt wurden, erzielte man wieder günstige Kassenabschlüsse, so dass die Vermögen der Kassen sich von Jahr zu Jahr recht erheblich steigerten."

13. Die Altersstruktur der Mitglieder übte in keinem unserer Modelle einen signifikanten Einfluss aus und wird daher in den Ergebnistabellen nicht explizit aufgeführt.

14. Nach der Daumenregel von Murray (2006) ist ein Instrument nicht als schwach zu bezeichnen, wenn die F-Statistik für den Koeffizienten der Instrumentvariable in der ersten Stufe der zweistufigen Instrumentvariablen (IV)-Schätzung Werte größer als 10 aufweist. In diesem Beispiel beträgt die zugehörige F-Statistik 11,36. 
Tabelle 2 Instrumentvariablen (IV)-Schätzung zur Erklärung der Krankentage pro Mitglied in preußischen und bayerischen Knappschaften, 1867-1907

\begin{tabular}{lcc}
\hline & IV, 2. Stufe & IV, 1.Stufe \\
\hline Krankenlohn pro Krankentag & $11,723(2,712)$ & - \\
Arbeitgeberanteil & $3,463(1,902)$ & $-0,021(-0,160)$ \\
Mitgliederzahl & $0,074(2,911)$ & $-0,004(-3,414)$ \\
Mitglieder pro Betrieb & $-0,188(-0,454)$ & $0,071(3,362)$ \\
Kohleproduktion & $-0,003(-1,201)$ & $0,008(6,386)$ \\
1887 und später & $-2,362(-2,354)$ & $0,297(13,691)$ \\
1905 und später & $1,853(3,721)$ & $-0,007(-0,212)$ \\
Jahr & $-0,123(-2,483)$ & $0,009(5,000)$ \\
Arbeitgeberbeitrag pro Mitglied & - & $0,004(4,379)$ \\
Konstante & $224,892(2,526)$ & $0,897(8,265)$ \\
Zahl der Beobachtungen & 2241 & 2241 \\
Untersuchte Knappschaften & 78 & 78 \\
\hline
\end{tabular}

t-Werte in Klammern. Für die Altersstruktur der Mitglieder wurde kontrolliert.

führte der Anstieg des Krankenlohns je Krankentag in der Tat zu einer deutlichen Steigerung der Krankentage pro Mitglied. Zweitens bewirkte auch die Erhöhung des Anteils der Arbeitgeberbeiträge eine Erhöhung der Krankheitstage je Mitglied. Das deutet darauf hin, dass es Bergleuten leichter fiel, sich krank zu melden, wenn ihr Krankenlohn zu einem geringeren Anteil von ihren Arbeitskollegen finanziert werden musste.

Drittens lässt sich der erwartete Größeneffekt beobachten: Mit steigender Mitgliederzahl der Knappschaften nahmen nach diesen Ergebnissen die Krankentage je Mitglied zu und somit wahrscheinlich auch die Fälle simulierter Krankheit. Gegenseitige soziale Kontrolle als Mechanismus zur Eindämmung von „Simulantentum“ funktionierte offenbar besser in kleineren Knappschaften. Dass dieser soziale Kontrollmechanismus auch noch in den großen und anonymen Krankenkassen des späten 20. Jahrhunderts funktionieren kann, zeigen Schnabel und Stephan (1993) anhand eines Firmenpanels. Der positive Zusammenhang zwischen Größe und Krankenstand zeigt sich in dieser Studie für die anhand der Belegschaftszahl gemessene Betriebsgröße - eine Variable, die der von uns ebenfalls berücksichtigten Determinanten „Mitglieder pro Betrieb“ entspricht. Arbeitnehmer sind und waren folglich tatsächlich in der Lage, spürbaren Einfluss auf das Verhalten ihrer Kollegen zu nehmen.

Viertens findet sich überraschenderweise kein signifikant positiver Zusammenhang zwischen dem Krankenstand und dem sektoralen Konjunkturverlauf, der durch die Kohlenproduktion in den Oberbergamtsbezirken gemessen wurde. Demzufolge missbrauchten weder die Arbeitgeber die Krankenversicherung in Krisenzeiten systematisch als implizite Arbeitslosenversicherung, noch simulierten Arbeitnehmer vorrangig in wirtschaftlichen Boomzeiten. Dieses Ergebnis steht im Gegensatz zu der auch heute noch oft vertretenen Hypothese, dass Arbeitnehmer während einer wirtschaftlichen Depression aus Angst vor Entlassung auf Simulation verzichten, in Zeiten des Booms dafür aber vermehrt krank feiern. Das Ergebnis verwundert besonders deshalb, weil vor dem Ersten Weltkrieg ein mit der heutigen Zeit vergleichbarer Kündigungsschutz nicht existierte und die Bergleute daher mehr als 


\section{Timothy W. Guinnane, Jochen Streb, Manuel Frondel und Christoph M. Schmidt}

heutige Arbeitnehmer Grund zur Befürchtung hatten, bei „Fehlverhalten“ während einer wirtschaftlichen Depression entlassen zu werden.

Fünftens deuten diese Resultate darauf hin, dass die Erhöhung und Verlängerung des Krankenlohns ab 1905 zu einem Anstieg der Krankheitstage je Mitglied führte. Demnach reagierten die Bergleute auf diese Verbesserung ihrer wirtschaftlichen Versorgung im Krankheitsfall mit einer erhöhten Zahl von Krankmeldungen. Der Effekt der Erhöhung der Lohnersatzleistungen ab 1887 ist allerdings signifikant negativ. Dieses Ergebnis könnte dadurch erklärt werden, dass die Knappschaftsverwaltungen auf die mit der Eingliederung in das Bismarcksche Sozialversicherungssystem verbundene sprunghafte Leistungserhöhung mit einer Verschärfung ihrer Kontrollmaßnahmen antworteten. Schließlich ergibt sich ein negativer Einfluss der Trendvariablen „Jahr“. In diesem Resultat könnte sich sowohl die Einführung von Unfallverhütungsvorschriften als auch das wachsende öffentliche Bewusstsein für die Schädlichkeit der Simulation widerspiegeln.

Zusammenfassend ist nach dieser Analyse ein positiver Zusammenhang zwischen Knappschaftsgröße und Krankenstand nicht von der Hand zu weisen. Knappschaftskrankenkassen mit mehr Mitgliedern verzeichneten demnach mehr Krankentage je Mitglied. Im Vergleich $\mathrm{zu}$ den Auswirkungen einer Erhöhung des Krankenlohns je Mitglied, die dann besonders hoch sind, wenn dieser zu einem hohen Anteil durch die Arbeitgeber finanziert wurde, ist dieser Effekt jedoch eher klein. Diese Schlussfolgerung würde erklären, warum die Knappschaften trotz all ihrer anhaltenden Klagen über die Simulanten die ihnen bereits durch das „Allgemeine Berggesetz für die preußischen Staaten“ im Jahr 1865 eingeräumte Möglichkeit, in ihrem Einzugsgebiet kleine und voneinander unabhängige Krankenkassen zu errichten, nicht nutzten.

Als wichtigstes empirisches Ergebnis ist festzuhalten, dass von der Höhe und Dauer des Krankenlohns je Krankentag ein starker Anreiz zur Simulation von Krankheit ausging: Je größer die Generosität der Lohnersatzleistung, desto höher war ceteris paribus der Krankenstand. Dieses Ergebnis steht im Gegensatz zu den Ausführungen von Scheil-Adlung und Sandner (2010), die solch einen Zusammenhang nicht beobachten, in ihrem aktuellen Vergleich verschiedener europäischer Krankenversicherungssysteme allerdings mit dem Problem unterschiedlicher gesetzlicher, ökonomischer und kultureller Rahmenbedingungen konfrontiert sind.

\subsection{Rheumatismus als "Simulantenkrankheit"}

Unsere Daten lassen noch einen weiteren Test des möglichen Vorliegens von Moral Hazard zu. In einer Welt ohne Simulation würde das Auftreten bestimmter Krankheiten oder Unfallarten nur durch medizinische Ursachen beeinflusst werden. Dazu zählen neben dem wellenartigen Auftreten von Epidemien auch die konkreten Arbeitsbedingungen der Bergleute. Ökonomische Faktoren hätten jedoch keinen messbaren Einfluss. Simulation kann man demnach möglicherweise dadurch identifizieren, dass man zeigt, dass die Anzahl von Fällen einer verhältnismäßig einfach zu „simulierenden“, weil diagnostisch schwer nachzuweisenden Krankheit statistisch signifikant von den jeweiligen ökonomischen Rahmenbedingungen abhängt. 
Tabelle 3 Unterschiede in der Zahl der Fälle von Rheumatismus und Prellungen, preußische Knappschaften, 1867-1884

\begin{tabular}{lccc}
\hline & $\begin{array}{c}\text { Alle } \\
\text { Knappschaften }\end{array}$ & $\begin{array}{c}\text { Stagnierende } \\
\text { Knappschaften }\end{array}$ & $\begin{array}{c}\text { Wachsende } \\
\text { Knappschaften }\end{array}$ \\
\hline Krankenlohn pro Krankentag & $0,182(2,069)$ & $0,327(2,341)$ & $0,111(0,817)$ \\
Arbeitgeberbeitrag & $0,043(0,717)$ & $0,210(2,014)$ & $0,040(0,417)$ \\
Mitgliederzahl & $-0,005(-0,940)$ & $-0,016(-1,394)$ & $-0,002(-0,316)$ \\
Mitglieder pro Betrieb & $-0,011(-0,596)$ & $0,019(0,577)$ & $-0,029(-1,054)$ \\
Braunkohleproduktion & $0,008(0,250)$ & $-0,061(-0,993)$ & $0,046(1,140)$ \\
Steinkohleproduktion & $0,001(0,424)$ & $0,001(0,167)$ & $0,002(0,597)$ \\
Konstante & $-0,050(-1,342)$ & $-0,177(-1,962)$ & $-0,018(-0,432)$ \\
Beobachtungen & 528 & 231 & 297 \\
Untersuchte Knappschaften & 56 & 19 & 37 \\
\hline
\end{tabular}

t-Werte in Klammern. Die abhängige Variable ist die Differenz zwischen der Zahl der Fälle von Rheumatismus und der Zahl der Fälle von Prellungen. Die Variable „Krankenlohn pro Krankentag“ wurde mit Hilfe des Instruments „Arbeitgeberbeitrag pro Mitglied“ geschätzt. Die Knappschaften wurden anhand des Medians der durchschnittlichen jährlichen Wachstumsrate der Mitgliederzahl von 1,8\% in stagnierende und wachsende Knappschaften unterteilt.

Die Zeitgenossen hatten insbesondere den Rheumatismus im Verdacht, der "Simulation“ Vorschub leisten zu können. ${ }^{15}$ Als Beleg für diese These wies Schlockow (1881) auf die großen Unterschiede in der Zahl rheumatischer Krankheiten in den verschiedenen Knappschaftskrankenkassen hin. Beispielsweise verzeichnete der Oberschlesische Knappschaftsverein nur 4,8 Fälle von Rheumatismus je 100 Mitglieder, während sich diese Zahl beim Clausthaler Knappschaftsverein auf 26,7 belief. Wenn die Zeitgenossen mit ihrer Einschätzung richtig lagen, dass Rheumatismus die „Simulantenkrankheit“ par excellence war, müsste sich auch auf statistischem Wege nachweisen lassen, dass die Zahl der diagnostizierten Rheumaerkrankungen verglichen mit einfach zu verifizierenden Prellungen in den Knappschaftskrankenkassen nicht nur von medizinischen Ursachen, sondern auch von ökonomischen Faktoren beeinflusst wurde.

Datenbeschränkungen erlauben hier lediglich eine Analyse der Situation in den preußischen Knappschaften zwischen 1867 und 1884. Tabelle 3 verdeutlicht, dass sich signifikante ökonomische Einflüsse nur für die Teilgruppe der stagnierenden Knappschaften nachweisen lassen. Erstens ist festzuhalten, dass die diagnostizierten Rheumaerkrankungen statistisch signifikant mit wachsendem Krankenlohn je Krankentag ansteigen. Bei der Interpretation dieses Resultats muss man jedoch

15. Der Mediziner Dr. Schlockow (1881, S. 160) der als Polizei- und Stadtphysicus in Breslau über die Gesundheitsverhältnisse des oberschlesischen Industriereviers detaillierte Studien durchgeführt hatte, führte hierzu aus: „Der Rheumatismus gehört zu den Krankheitsbezeichnungen, denen in diagnostischer und statistischer Beziehung mit einer gewissen Vorsicht begegnet werden muss. Abgesehen davon, dass die Intensität der Krankheit eine sehr verschiedene sein kann und dass in unserem Material die vollständig von einander zu trennenden Formen des fieberhaften Gelenkrheumatismus und des fieberlosen leichten Muskelrheumatismus nicht auseinander gehalten sind, gibt es keinen Krankheitsnamen, hinter dem sich der Mangel einer eingehenden ärztlichen Diagnose und in Hilfskassenvereinen bei Neigung zur Simulation der Mangel einer Krankheit überhaupt lieber und leichter verbirgt." 


\section{Timothy W. Guinnane, Jochen Streb, Manuel Frondel und Christoph M. Schmidt}

vorsichtig sein: Es könnte entweder bedeuten, dass sich mehr, tatsächlich am Rheuma erkrankte Bergleute bei wachsendem Krankenlohn eine Erholungszeit leisten konnten, oder, dass mehr Bergleute bei wachsendem Krankenlohn Rheuma simulierten.

Zweitens steigen für die stagnierenden Krankenkassen die Rheumafälle auch mit zunehmendem Anteil der Arbeitgeberbeiträge an. Auch dieser Befund bestätigt die Ergebnisse aus Tabelle 2. Allerdings hat, drittens, die Knappschaftsgröße keinen signifikanten Einfluss auf die Zahl der Rheumaerkrankungen. Möglicherweise versagte der soziale Kontrollmechanismus von kleinen und überschaubaren Knappschaftskrankenkassen im Falle von Rheumatismus deshalb, weil die Bergleute - und ebenso die Ärzte - nicht dazu in der Lage waren, einzuschätzen, ob ihr Arbeitskollege tatsächlich daran litt oder diese Krankheit nur simulierte.

\section{Zusammenfassung und Schlussfolgerung}

Die wirtschaftshistorischen Untersuchungen von Guinnane und Streb (2011) über die Knappschafts-Krankenversicherungen haben gezeigt, dass schon im 19. Jahrhundert ein signifikant positiver Zusammenhang zwischen der Generosität des Krankenlohns je Krankentag und dem Krankenstand bestand. Als Politikempfehlung für die aktuelle Situation, die sich aus den historischen Erfahrungen der Knappschaften ableiten lässt, mag es deshalb zur Senkung der mit den Krankenversicherungsbeiträgen verbundenen Lohnnebenkosten naheliegen, eine Kürzung dieser Lohnersatzleistung vorzunehmen. Um sicherzustellen, dass sich die tatsächlich Kranken die Zeit nehmen können, ihre Krankheit vollständig auszukurieren, sollte man dabei allerdings davon absehen, eine pauschale Reduktion der Lohnfortzahlung von derzeit 100 Prozent auf beispielsweise 70 bis 80 Prozent des Nettolohns vorzunehmen, wie dies die Regierung unter Bundeskanzler Kohl im Jahr 1995 tat und was von der Regierung Schröder 1999 wieder rückgängig gemacht wurde. Sinnvoller erscheint die Wiedereinführung von Karenztagen, da diese Form der Kürzung der Lohnersatzleistung die wirtschaftliche Absicherung des Patienten und seiner Familie bei schwerer und langwieriger Krankheit nicht beeinträchtigt.

Die hier diskutierte wirtschaftshistorische Analyse unterstützt auch das von Schnabel und Stephan (1993) für das späte 20. Jahrhundert nachgewiesene Ergebnis, dass Arbeitnehmer tatsächlich dazu in der Lage sind, ihre Kollegen durch die Ausübung von sozialem Druck vom Missbrauch der Krankenversicherung abzuhalten. Es spricht daher einiges dafür, ein entsprechendes Problembewusstsein in den Betrieben dadurch zu schaffen, dass man den Arbeitnehmern durch regelmäßige Information vor Augen führt, dass „Simulation“ nicht nur das anonyme System schädigt, sondern zu höheren Krankenversicherungsbeiträgen der Kollegen führt und überdies über die Steigerung der Lohnnebenkosten Arbeitsplätze unsicherer macht.

In diesem Aufsatz haben wir aufgezeigt, dass wirtschaftshistorische Betrachtungen, in diesem Fall des Krankenstands der Knappschaftskrankenkassen des späten 19. und frühen 20. Jahrhunderts, aus methodischer Sicht wertvolle Beiträge zur gesundheitsökonomischen Literatur und zur aktuellen Politikdebatte leisten können. So eignet sich diese wirtschaftshistorische Perspektive besonders gut zur Analyse von aktuell höchst relevanten Fragestellungen bezüglich 
der Organisation unseres heutigen Krankenversicherungssystems, da die Knappschaften einen weitaus größeren geschäftspolitischen Handlungsspielraum besaßen als die heutigen Krankenkassen, obwohl die Knappschaften in der Formationsphase des deutschen Wohlfahrtsstaats - ebenso wie die heutigen Krankenkassen - unter einem gemeinsamen Ordnungsrahmen agierten.

Die aus diesem geschäftspolitischen Handlungsspielraum resultierende starke Variation der versicherungsinternen Governance, aber auch die Veränderung der äußeren Rahmenbedingungen während einer langen historischen Phase, welche sich in dem hier diskutierten Paneldatenmaterial widerspiegeln, ist zur Gewinnung empirischer Einsichten unverzichtbar. So steigert die gegenüber den gegenwärtigen Krankenkassen weitaus höhere Variabilität in der Höhe und Dauer der Lohnfortzahlung der Knappschaften, welche als die zentralen versicherungsökonomischen Einflussgrößen anzusehen sind, die Aussagekraft der auf Basis von panelökonometrischen Modellen gewonnenen empirischen Resultate erheblich.

Aktuelle Querschnitts- und Paneldaten auf nationaler Ebene können hingegen keine derartige Variation der Umstände anbieten, während international vergleichende Daten von erheblichen Problemen unbeobachtbarer Heterogenität geplagt sein dürften. Darüber hinaus hat das wirtschaftshistorische Datenmaterial den Vorteil, dass die Lebensspannen der in den Knappschaften Versicherten abgeschlossen sind, wohingegen aktuelles Datenmaterial oftmals den Nachteil hat, lediglich Momentaufnahmen von Krankenversicherungen zeichnen zu können, und daher potentielle Probleme infolge der Alterung ihrer Mitglieder nicht unbedingt erkannt würden. Wenngleich das historische Datenmaterial selbstverständlich ebenfalls nicht ohne Identifikationsannahmen analysiert werden kann, dürften diese von weit weniger drastischer Natur sein, als es in Studien über gegenwärtige Versicherungssysteme möglich wäre.

Der große Vorteil einer wirtschaftshistorischen Betrachtung des deutschen Sozialversicherungssystems vor dem Ersten Weltkrieg, der in einer starken Variation der Geschäftspolitiken vergleichsweise unabhängiger Sozialversicherungsträger bei zugleich homogenen äußeren Rahmenbedingungen besteht, kann in weitaus größerem Maße zur Beantwortung von aktuellen Fragestellungen genutzt werden als allein zur Analyse von Moral Hazard bei Krankenversicherungen.

Beispielsweise untersucht (Jopp 2011a, 2011b), wie die umlagefinanzierten knappschaftlichen Invaliditätsversicherungen mit dem Problem der Überalterung ihrer Mitglieder zu Rande kamen, und liefert hierdurch wertvolle Hinweise für die Beurteilung der durch den demographischen Wandel drohenden Gefahren, die auf das gegenwärtige deutsche Rentenversicherungssystem zukommen. Guinnane und Streb widmen sich in einem gerade begonnenen Projekt der Frage, wie sich die Ausgestaltung der Gefahrentarife der in den 1880er Jahren gegründeten Berufsgenossenschaften auf die Unfallverhütung und damit auf die Unfallhäufigkeit in den Unternehmen der Eisen- und Stahlindustrie auswirkten. Dies sind lediglich zwei Beispiele dafür, dass die Wirtschaftsgeschichte des deutschen Sozialversicherungssystems das ideale - und bislang vernachlässigte „Laboratorium“ für empirische Studien zur Wirkung sozialökonomischer Instrumente bietet. 


\section{Timothy W. Guinnane, Jochen Streb, Manuel Frondel und Christoph M. Schmidt}

\section{Literaturverzeichnis}

Arndt, A. (1894), Bergbau und Bergbaupolitik. Hirschfeld, Leipzig.

Barmby, T.A. und J.G. Treble (1991), Betriebliche Fehlzeiten und Arbeitsverträge, Mitteilungen aus der Arbeitsmarkt- und Berufsforschung 3/91, 595-604.

Bartels, C. et al. (2009), Vergangenheit und Zukunft sozialer Sicherungssysteme am Beispiel der Bundesknappschaft und ihrer Nachfolger. Ein Forschungsprojekt der LeibnizGesellschaft, Jahrbuch für Wirtschaftsgeschichte 2009/2, 195-217.

Bluma, L., S. Schulz und J. Streb (2010), Prinzipal-Agenten-Probleme in der knappschaftlichen Krankenversicherung: Die Bekämpfung des „Simulantentums“ durch Anreize und Kontrolle, Vierteljahrschrift für Sozial- und Wirtschaftsgeschichte 97, 310-334.

Borowiak, F. und T. Taubert (1997), Das Rückkehrgespräch: Ein Instrument gesundheitsgerechter Personalführung, Personalführung 11/97, 1086-1091.

Boss, A. (2000), Lohnfortzahlung und Krankenstand, Zeitschrift für Wirtschaftspolitik 49, 27 50.

Bülow, W. (1905), Das Knappschaftswesen im Ruhrkohlenbezirk bis zum allgemeinen preußischen Berggesetz vom 24. Juni 1865. Universitäts-Dissertation, Tübingen.

Fahr, R. und B. Frick (2007), On the Inverse Relationship between Unemployment and Absenteeism: Evidence from Natural Experiments and Worker Heterogeneity, IZA Discussion Paper 3171.

Guinnane, T.W. und J. Streb (2011), Moral Hazard in a Mutual Health-Insurance System: German Knappschaften, 1867-1914, Journal of Economic History 71, 70-104.

Hennock, E.P. (2007), The Origin of the Welfare State in England and Germany, 1850-1914: social policies compared. Cambridge University Press, Cambridge.

Iverson, D.C. und R. Krause (2007), Produktivitätsräuber Präsentismus, Personalmanagement $12 / 2007,46-48$.

Jopp, T. (2010), Ein risikoreiches Geschäft? Internes und externes Wachstum als risikopolitische Instrumente im preußischen Knappschaftswesen, 1854-1923, in: C. Bartels (Hrsg.), Berufliches Risiko und Soziale Sicherheit: Beiträge zur Tagung,Vergangenheit und Zukunft sozialer Sicherungssysteme am Beispiel der Bundesknappschaft und ihrer Nachfolger' im Deutschen Bergbau-Museum Bochum, 8. und 9. Oktober 2009. Deutsches Bergbaumuseum, Bochum, 189-224.

Jopp, T. (2011a), Old Times, Better Times? German Miners' Knappschaften, Pay-as-you-go Pensions, and Implicit Rates of Return, 1854-1913, Business History 53, 1018-1043.

Jopp, T. (2011b), The Hazard of Merger by Absorption - Why Some Knappschaften Merged and Others Did Not: 1861-1920, Zeitschrift für Unternehmensgeschichte/Journal of Business History 56, 75-101.

Lauf, U. (2006), Die deutschen Knappschaftsvereine (1854-1923): Ein altes Sozialsystem im Rückblick, Der Anschnitt 58, 264-294.

Menzel, E. (1989), Bergbau-Medizin einst und jetzt: Entwicklung des bergmännischen Gesundheitswesens unter Einschluß der Kranken- und Unfallversicherung. Erich Schmidt, Berlin.

Murray, J. (2005), Worker Absenteeism under Voluntary and Compulsory Sickness Insurance: Continental Europe, 1885-1908, Research in Economic History 23, 177-207.

Murray, M.P. (2006), Avoiding Invalid Instruments and Coping with Weak Instruments. Journal of Economic Perspectives 20, 111-132.

Olesch, G. (1993), Pragmatische Maßnahmen zur Reduzierung des Krankenstandes, Personal: Mensch und Arbeit im Betrieb 45, 570-574.

Riley, J.C. (1997), Sick, not Dead: the Health of British Workingmen during the Mortality Decline. Johns Hopkins University Press, Baltimore, Md.

Scheil-Adlung, X. und L. Sandner (2010), Evidence on Paid Sick Leave: Observations in Times of Crisis, Intereconomics: Review of European Economic Policy 45, 313-321. 


\title{
Krank oder Simulant?
}

Schnabel, C. und G. Stephan (1993), Determinanten des Krankenstandes: Eine Untersuchung mit Betriebs- und Zeitreihendaten, Jahrbuch für Sozialwissenschaft 44, 132-147.

Schlockow, I. (1881), Die Gesundheitspflege und medizinische Statistik beim Preußischen Bergbau. C. Heymann, Berlin.

Spies, S. und H. Beigel (1996), Einer fehlt, und jeder braucht ihn: Wie Opel die Abwesenheit senkt. Ueberreuter, Wien.

\begin{abstract}
Economic-historical findings attest that already in the nineteenth century the economic implications of Moral Hazard in health insurance were very well understood and that attempts were made to overcome them with the help of various incentive and control mechanisms. This article shows that the health insurance schemes run by the historical miners' guilds, by reason of their monopoly in the sector and their relatively wide room for maneuver in the politics of the business, are particularly well suited to an empirical analysis of the problems connected with Moral Hazard. We conclude that the economichistorical perspective here adopted can be extended beyond the analysis of Moral Hazard in health insurance to answer other present-day questions. The most important finding of an empirical analysis based on data concerning historical miners' guilds is that the level and duration of daily sickness pay created a strong incentive to feign illness.
\end{abstract}

